\title{
INSTRUMENTATION \& TECHNIQUES An inexpensive infusion pump suitable for chronic administration of liquids to restrained or unrestrained animals
}

\author{
D. H. VANDERCAR \\ University of South Florida, Tampa, Florida 33620
}

\begin{abstract}
A self-contained, inexpensive, disposable infusion pump is described for chronic or acute administration of solutions. The pump consists of a modified disposable syringe that is capable of delivering liquids at rates which may be varied. Pressure resulting from a chemical reaction within a chamber propels a rubber piston, which in turn expels liquids at a uniform rate.
\end{abstract}

The chronic infusion of anticoagulants, anesthetics, and other drugs to unrestrained animals has long been recognized as a valuable research technique. Typically, the administration of liquids to freely moving animals has required the connecting of an indwelling catheter to a motor-driven infusion pump via a liquid swivel joint. Although this technique has been useful in many applications, it has several disadvantages: Motor-driven infusion pumps and swivel joints are expensive to purchase or difficult to construct, and the utilization of commercially available infusion pumps restricts subject movement.

The device shown in Figure 1 is a modified plastic disposable syringe (10 cc B-D Plastipack) capable of delivering a total of $7 \mathrm{cc}$ of solution at rates that may be varied from less than $.2 \mathrm{cc} / \mathrm{h}$ to greater than $2 \mathrm{cc} / \mathrm{h}$. This device can be assembled in $5 \mathrm{~min}$ with materials costing less than 25 cents.

Figure 1 depicts the fully assembled unit. Parts $C$ and $G$ are two rubber pistons that have been removed from their shafts. Piston $G$ is inserted in its normal forwardfacing position; Piston $C$ is inserted in the reverse manner, leaving a small space (E) intervening. Chamber $\mathrm{H}$ contains the solution to be delivered. A small piece of cotton $(F)$, moistened with three drops of water, is inserted into the cavity of Piston G. Piston $\mathrm{C}$ contains a pellet of calcium carbide ${ }^{1}$ (B), approximately $4 \mathrm{~mm}$ in diam. Covering the opening to Piston $C$, and securing the pellet, is a circular piece of paper adhesive (D). The adhesive retainer is perforated at its center with a 26-gauge needle. The pressure in Chamber $\mathrm{E}$ is developed by the production of acetylene gas, which results from the interaction of water vapor with the calcium carbide pellet. The rate at which acetylene is produced within Chamber $\mathrm{E}$ is a function of the number of holes that

Reprint requests should be mailed to the author at the Psychology Department, University of South Florida, Tampa, Florida 33620. have been introduced into the paper adhesive tape. As pressure in Chamber E builds, it slowly forces Piston G forward, thus expelling the liquid at a constant rate. Piston $\mathrm{C}$ is prohibited from being expelled from the rear of the pump by a small ridge (A) which is found at the rear of most disposable syringes.

The application of the paper adhesive tape (cut slightly smaller than the outside diameter of the piston) is facilitated if care is taken to remove any silicone grease present on the surface of Piston C. Additionally, the tape prevents the pellet of calcium carbide from coming into physical contact with the moist cotton. This allows the pump to function properly in all positions. After Pistons $G$ and $C$ have been inserted, liquid is introduced to the pump from the front end with the aid of a syringe and a needle.

When loading the pump, care should be taken to exclude air bubbles from Section $H$. Once prepared in this manner, the pump will immediately begin expelling liquid.

A cautionary note: In the event that the cannula becomes obstructed, or if the pump is not dismantled after all liquid is expelled, it is possible that Piston C will become dislodged due to an overabundance of pressure, releasing acetylene gas into the laboratory.

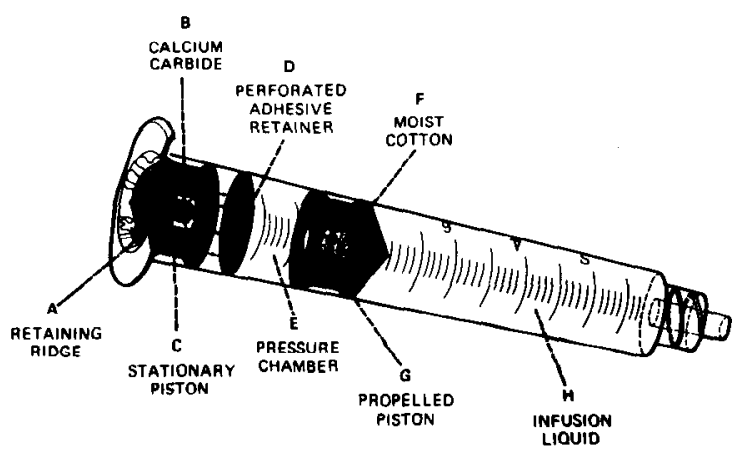

Figure 1. Illustration of gas-propelled infusion pump. 


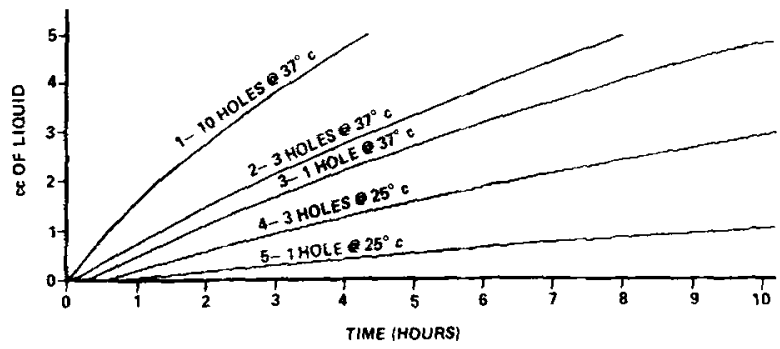

Figure 2. Rates of infusion of liquids from a $10-\mathrm{cc}$ infusion pump under five different conditions.

Although acetylene gas is used as a propellant, a standard qualitative test (Reid, 1966) revealed no detectable traces of acetylene in the expelled liquid.

Figure 2 depicts measurements of rates at which liquids are expelled from a 10-cc syringe under five different conditions. A modified dew meter was used to measure the amount of water, in weight, that fell into a plastic cup over a 10 -h period. Curves 1,2 , and 3 represent the rates of delivery of water when the device was at body temperature $\left(37^{\circ} \mathrm{C}\right)$ and pumping against a pressure equal to $100 \mathrm{~mm}$ of mercury. Curve 1 illustrates the rate of flow of liquid obtained with 10 holes in the adhesive retainer. Curves 2 and 3 illustrate slower rates of infusion when three holes and one hole were introduced, respectively, into the adhesive retainer. Curves 4 and 5 represent data collected in a manner identical to the data of Curves 1,2 , and 3 , with the exception that the syringe pump was maintained at room temperature $\left(25^{\circ} \mathrm{C}\right)$. The slower rates of delivery illustrated in the latter two curves reflect the effect of temperature upon the chemical reaction. Figure 2 indicates that the amount of liquid delivered over time is reasonably linear.

Although the degree of accuracy and repeatability with which liquids can be administered does not reach that of commercially available motor-driven infusion pumps, this disadvantage is, to a rather large extent, offset by economy as well as the greater freedom of movement afforded to the experimental animal.

This pump may be used with either acute or chronic preparations. For subjects as large as rabbits or primates, the pump may be surgically stitched to the back of the subject or, alternatively, contained within a vest which is strapped to the animal's back.

\section{REFERENCE}

ReID. K. F. Properties and reactions of bonds in organic molecules. New York: American Elsevier, 1966. Pp. 268.

\section{NOTE}

1. Calcium carbide may be obtained in 1-lb quantities ("small lumps") from Matheson, Coleman, and Bell, manufacturing chemists, 2909 Highland Avenue, Norwood, Ohio 45212.

(Accepted for publication October 21 1976.) 